\title{
REDUCTION OF STRUCTURE FOR TORSORS OVER SEMILOCAL RINGS
}

\author{
V. CHERNOUSOV, P. GILLE, AND Z. REICHSTEIN
}

\begin{abstract}
Let $G$ be a reductive affine group scheme defined over a semilocal ring $k$. Assume that either $G$ is semisimple or $k$ is normal and noetherian. We show that $G$ has a finite $k$-subgroup $S$ such that the natural map $H^{1}(R, S) \rightarrow H^{1}(R, G)$ is surjective for every semilocal ring $R$ containing $k$. In other words, $G$-torsors over $\operatorname{Spec}(R)$ admit reduction of structure to $S$. We also show that the natural map $H^{1}(X, S) \rightarrow H^{1}(X, G)$ is surjective in several other contexts, under suitable assumptions on the base ring $k$, the scheme $X / k$ and the group scheme $G / k$. These results have already been used to study loop algebras and essential dimension of connected algebraic groups in prime characteristic. Additional applications are presented at the end of this paper.
\end{abstract}

\section{Contents}

\section{Introduction}

2. Preliminaries

3. A first step towards the proof of Theorem 1.2

4. Proof of Theorem 1.2(a) and (b)

5. Toral torsors and a theorem of Grothendieck

6. Proof of Theorem 1.2(c)

7. Proof of Theorem 1.3 and an application

8. Torsors on affine spaces

References

\section{INTRODUCTION}

Let $G$ be a linear algebraic group defined over a field $k$. In [CGR] we showed that, under mild assumptions on $G$ and $k, G$ has a finite $k$-subgroup $S$ such that every $G$-torsor over a field $K / k$ admits reduction of structure

1991 Mathematics Subject Classification. 11E72, 14L15, 20G10.

Key words and phrases. Linear algebraic group, group scheme, torsor, non-abelian cohomology.

V. Chernousov was partially supported by the Canada Research Chairs Program and an NSERC research grant.

Z. Reichstein was partially supported by an NSERC research grant. 
to $S$, i.e., the natural map of Galois cohomology sets $H^{1}(K, S) \rightarrow H^{1}(K, G)$ is surjective. In several subsequent applications, a more general version of this result was needed, with the field $k$ replaced by a base ring, the group $G$ by a reductive group scheme over $k$ and the field $K / k$ by a $k$-scheme $X$. The goal of this paper is to extend the main result of [CGR] to this more general setting.

All schemes in this paper will be assumed to be locally noetherian. Of particular interest to us will be $k$-schemes $X$ satisfying the following condition:

$$
\operatorname{Pic}\left(X^{\prime}\right)=0 \text { for every generalized Galois cover } X^{\prime} / X \text {. }
$$

Here by a generalized Galois cover $X^{\prime} \rightarrow X$ we mean a $\Gamma$-torsor, for some twisted finite constant group scheme $\Gamma$ defined over $X$. In other words, $\Gamma={ }_{a} C$, where $C$ is a finite constant group scheme over $X$ and $[a] \in$ $H^{1}(X, \operatorname{Aut}(C))$. (The term "Galois cover" is usually reserved for the case where $\Gamma$ is itself a finite constant group scheme.) The class of schemes satisfying condition (1.1) includes, in particular, affine schemes of the form $X=\operatorname{Spec}(R)$, where $R$ is a semilocal ring containing $k$. If $K$ is a $k$-field of characteristic 0 , we can also take $R$ to be a polynomial ring $K\left[x_{1}, \ldots, x_{n}\right]$ (see 8 ) or a Laurent polynomial ring $K\left[x_{1}^{ \pm 1}, \ldots, x_{n}^{ \pm 1}\right]$ (see Remark 8.3).

We are now ready to state the main results of this paper. Recall that an $X$-group $T$ of multiplicative type is called isotrivial if $T \times_{X} X^{\prime}$ is split for some finite étale surjective map $X^{\prime} \rightarrow X$. For the definition and basic properties of groups of multiplicative type, we refer the reader to [SGA3, $\mathrm{X}]$.

1.2. Theorem. Let $k$ be a commutative base ring and $G$ be a smooth affine group scheme over $k$ whose connected component $G^{0}$ is reductive. Assume further that one of the following holds:

(a) $k$ is an algebraically closed field, or

(b) $k=\mathbb{Z}, G^{0}$ is a split Chevalley group, and the order of the Weyl group of the geometric fiber $G_{\bar{s}}$ is independent of $s \in \operatorname{Spec}(\mathbb{Z})$, or

(c) $k$ is a semilocal ring, $G$ is connected, and the radical torus $\operatorname{rad}(G)$ is isotrivial.

Then there exist a maximal torus $T \subset G$ defined over $k$ and a finite $k$ subgroup $S \subset N_{G}(T)$, such that

(1) $S$ is an extension of a twisted constant group scheme by a finite $k$-group of multiplicative type,

(2) the natural map $H^{1}(X, S) \longrightarrow H^{1}\left(X, N_{G}(T)\right)$ is surjective for any scheme $X / k$ satisfying condition (1.1).

Of course, if $G$ is connected then (a) is a special case of (c). Note also that in case $(b)$ we can take $G$ to be the automorphism group Aut $\left(G_{0}\right)$ of some semisimple Chevalley group scheme $G_{0}$. In this case the cohomology set 
$H^{1}(X, G)$ classifies the semisimple group schemes over $X$, which are étale locally isomorphic to $G_{0} \times_{\mathbb{Z}} X$.

Combining Theorem 1.2(c) with Grothendieck's existence theorem for maximal tori (reproduced as Theorem 5.2 below), we obtain the following stronger result in case (c); cf. 87

1.3. Theorem. Let $k, G$ and $S$ be as in Theorem 1.2(c). Then the map $H^{1}(R, S) \rightarrow H^{1}(R, G)$ is surjective for any semilocal ring $R / k$.

Note that the assumption on the radical of $G$ is superfluous if $G$ is a semisimple group scheme or if $k$ is normal and noetherian, because all tori defined over such rings are isotrivial; see [SGA3, X.5.16].

The symbol $H^{1}(X, G)$ in the statements of Theorems 1.2 and 1.3 denotes the flat cohomology set, which classifies $G$-torsors over $X$; see 92 . If $G$ is smooth then every $G$-torsor over $X$ is also smooth and is trivialized by an étale covering [M, III.4]. So in this case the natural map $H_{e ́ t}^{1}(X, G) \rightarrow$ $H^{1}(X, G)$ is bijective, and we may replace $H^{1}(X, G)$ by $H_{e ́ t}^{1}(X, G)$.

In particular, suppose that $k$ is an algebraically closed field and $G / k$ and $S / k$ are as in Theorem [1.2(a). If $K$ is a perfect field containing $k$ and $K_{s}$ is the separable closure of $K$ then

$$
H^{1}(K, G)=H^{1}\left(\operatorname{Gal}\left(K_{s} / K\right), G\left(K_{s}\right)\right)
$$

and

$$
H^{1}(K, S)=H^{1}\left(\operatorname{Gal}\left(K_{s} / K\right), S\left(K_{s}\right)\right) .
$$

In other words, in this situation the flat cohomology sets appearing in the statement of Theorem 1.2 (a) can be replaced by Galois cohomology. Moreover, since $S$ is finite, $S(k)=S\left(K_{s}\right)$ (with $\operatorname{Gal}\left(K_{s} / K\right)$ acting trivially on both sides) and hence,

$$
H^{1}\left(\operatorname{Gal}\left(K_{s} / K\right), S(k)\right)=H^{1}\left(\operatorname{Gal}\left(K_{s} / K\right), S\left(K_{s}\right)\right) .
$$

Thus in this setting Theorem 1.2(a) implies the following characteristic-free result about Galois cohomology. The assertion about $|S|:=\operatorname{dim}_{k} k[S]$ is immediate from the construction of $S$ in 4 .

1.4. Corollary. Let $G$ be a linear algebraic group defined over an algebraically closed field $k$, whose connected component $G^{0}$ is reductive. Then there exists a finite $k$-subgroup $S$ of $G$, such that every prime factor of $|S|$ divides the order of the Weyl group $W(G)$, and the map

$$
H^{1}\left(\operatorname{Gal}\left(K_{s} / K\right), S(k)\right) \rightarrow H^{1}\left(\operatorname{Gal}\left(K_{s} / K\right), G\left(K_{s}\right)\right)
$$

is surjective for any perfect field $K / k$.

Corollary 1.4 generalizes [CGR, Theorem 1.1(a)], which yields the same conclusion if $\operatorname{char}(k)=0$. This corollary has been used to study essential dimension of connected algebraic groups in positive characteristic in GR. An application of Theorem 1.2 to the study of loop algebras can be found in GP] cf. Remark 8.3. We will give additional applications in $\S \S 7$ and 8 . 
A further application of Theorem 1.2 will appear in the forthcoming paper CGP].

We are grateful to M. Florence for pointing out a mistake in the proof of CGR, Theorem 1.1(a)]. This mistake is corrected in the course of the proof of Lemma 3.2. For details, see Remark 4.1.

\section{PReliminaries}

We begin by recalling some known facts about affine group schemes $G$ of finite type over an arbitrary base scheme $X$.

A pseudo G-torsor (formellement principal homogène, in [SGA3]) E over $X$ is an $X$-scheme equipped with a right action of $G$ such that the mapping $E \times{ }_{X} G \rightarrow E \times{ }_{X} E$ given by $(x, g) \mapsto(x, x . g)$ is an isomorphism; see [SGA3, IV.5.1]. A pseudo $G$-torsor $E$ is a G-torsor (fibré principal homogène) if it is locally trivial in the fppf topology, i.e., if there exists a faithfully flat morphism $X^{\prime} \rightarrow X$, locally of finite type, such that $E \times{ }_{X} X^{\prime} \cong G \times_{X} X^{\prime}$. Here, as usual, the acronym fppf stands for "fidèlement plate de présentation finie" or "faithfully flat and finitely presented".

For such a covering $X^{\prime} \rightarrow X$, we define

$$
Z^{1}\left(X^{\prime} / X, G\right):=\left\{g \in G\left(X^{\prime} \times_{X} X^{\prime}\right) \mid p_{1,2}^{*}(g) p_{2,3}^{*}(g)=p_{1,3}^{*}(g)\right\}
$$

and

$$
H^{1}\left(X^{\prime} / X, G\right):=Z^{1}\left(X^{\prime} / X, G\right) / G\left(X^{\prime}\right),
$$

where $G\left(X^{\prime}\right)$ acts on $Z^{1}\left(X^{\prime} / X, G\right)$ by $g \cdot z=p_{1}^{*}(g) z p_{2}^{*}(g)^{-1}$; see [K, Chapter III]. Here $p_{i, j}: X^{\prime} \times_{X} X^{\prime} \times_{X} X^{\prime} \rightarrow X^{\prime} \times_{X} X^{\prime}$ is the projection

$$
\left(x_{1}^{\prime}, x_{2}^{\prime}, x_{3}^{\prime}\right) \rightarrow\left(x_{i}^{\prime}, x_{j}^{\prime}\right)
$$

and $p_{1,2}^{*}(g), p_{2,3}^{*}(g), p_{1,3}^{*}(g)$ are viewed as elements of $G\left(X^{\prime} \times_{X} X^{\prime} \times_{X} X^{\prime}\right)$. The pointed set $H^{1}\left(X^{\prime} / X, G\right)$ classifies $G$-torsors over $X$ which are trivialized by the base change $X^{\prime} / X$, i.e., $G$-torsors $E$ satisfying

$$
E \times_{X} X^{\prime} \stackrel{\sim}{\longrightarrow} G \times_{X} X^{\prime}
$$

see [M, III.4, page 120]. We now define

$$
H^{1}(X, G):=\underset{X^{\prime}}{\lim _{1}} H^{1}\left(X^{\prime} / X, G\right),
$$

where the limit is taken over all coverings $X^{\prime} / X$ in the $f p p f$ topology. The pointed set $H^{1}(X, G)$ classifies $G$-torsors over $X$.

If $P$ is a $G$-torsor over $X$, we denote by ${ }^{P} G$ the associated twisted $X$ group scheme; it is the twisted inner form of $G$ and can be defined as the scheme of $G$-automorphisms of $P$. We then have a canonical bijection (the "torsion" map)

$$
H^{1}(X, G) \stackrel{\simeq}{\rightarrow} H^{1}\left(X,{ }^{P} G\right)
$$

mapping a $G$-torsor $Q$ to the scheme $\underline{\operatorname{Isom}}_{G}(P, Q)$ of $G$-isomorphisms of $P$ into $Q$; see Gir, III.2.6]. In particular, the torsion map takes $P$ to the trivial ${ }^{P} G$-torsor. 
We say that $G$ is connected if the fiber $G_{x}$ is connected for any point $x \in X$. Here we view $G_{x}=G \times_{X} \operatorname{Spec}(\kappa(x))$ as an algebraic group over the residue field $\kappa(x)$ of $x$. If $G / X$ is smooth, then $G$ contains a unique maximal open connected normal subgroup defined over $X$; SGA3. $\mathrm{VI}_{B}$, Thm. 3.10]. As usual, we will denote this subgroup by $G^{0} / X$ and refer to it as the connected component of $G$. Note that $G^{0}$ is smooth over $X$ and it is a closed subgroup of $G$; in particular, it is affine over $X$.

We say that $G / X$ is reductive if it is smooth and all of its geometric fibers $G_{\bar{x}}$ are (connected) reductive groups [SGA3, XIX.2.7]. A subgroup $T / X$ of $G / X$ is a maximal torus if it is an $X$-torus and all of its geometric fibers are maximal tori [SGA3, XII.1.3]. The radical torus $\operatorname{rad}(G)$ of $G$ is the unique maximal torus of the center of $G$ [SGA3, XXII.4.3.6].

Similarly a subgroup $B / X$ of a reductive group scheme $G / X$ is a Borel subgroup if it is smooth and finitely presented and all of its geometric fibers are Borel subgroups [SGA3, XXII.5.2.3].

We refer to [SGA3, XXII.1] for the definitions of split group schemes and to [SGA3, XXIV.3] for the definition of the Dynkin scheme of $G$ and quasi-split reductive group schemes.

Let $G$ be a split adjoint semisimple group over $X, T$ a maximal split torus in $G$ defined over $X, B$ a Borel subgroup containing $T$ and $D / X$ the corresponding Dynkin scheme of $G$. Following [SGA3, XXIV.3.5], we will denote the group scheme representing the functor of automorphisms of $D$ (as a Dynkin scheme) by $\operatorname{Aut}_{D y n}(D)$. By [SGA3, XXIV, Théorème 1.3 and $3.6]$

$$
\operatorname{Aut}(G)=G \rtimes \operatorname{Aut}_{D y n}(D) .
$$

Moreover, there exists a canonical splitting

$$
h: \operatorname{Aut}_{D y n}(D) \rightarrow \operatorname{Aut}(G)
$$

such that the image of $h$ preserves $T$ and $B$. Every quasi-split adjoint group scheme $G^{\prime}$ of the same type as $G$ is $X$-isomorphic to the twist ${ }_{h_{*}(a)} G$ of $G$ for some cocycle

$$
a \in Z_{e ́ t}^{1}\left(X, \operatorname{Aut}_{D y n}(D)\right)
$$

\section{A first step towards the Proof of Theorem 1.2}

The purpose of this section is to prove the following proposition, which will play a key role in the proof of Theorem 1.2 .

3.1. Proposition. Let $k$ be a commutative base ring and

$$
1 \rightarrow T \rightarrow N \stackrel{p}{\rightarrow} W \rightarrow 1
$$

be an exact sequence of smooth group schemes defined over $k$, where $T$ is an isotrivial torus, split by a Galois extension $k^{\prime} / k$ of degree $d$, and $W$ is a twisted finite constant group of order $n$. Suppose $N$ has a finite $k$-subgroup $S^{\prime}$ such that $p\left(S^{\prime}\right)=W$. Then there exists a finite $k$-subgroup $S \subset G$ 
containing $S^{\prime}$ such that the natural map $H^{1}(X, S) \rightarrow H^{1}(X, N)$ is surjective for any $k$-scheme $X$ satisfying condition (1.1).

Moreover, we can take $S$ to be the subgroup of $N$ generated by $S^{\prime}$ and $\phi_{m}^{-1}\left(S^{\prime} \cap T\right)$, where $m=n d$ and $\phi_{m}: T \rightarrow T$ is the map taking $t \in T$ to $t^{m}$.

Proof. Denote by $q: S \rightarrow W$ and $q^{\prime}: S^{\prime} \rightarrow W$ the restrictions of the projection $p: N \rightarrow W$ to $S$ and $S^{\prime}$, and by $\mu=S \cap T$ and $\mu^{\prime}=S^{\prime} \cap T$ the kernels of these maps, respectively. Let $X$ be a $k$-scheme satisfying condition (1.1) on Picard groups. We will prove the surjectivity of $H^{1}(X, S) \rightarrow H^{1}(X, N)$ fiberwise, with respect to the mapping $p_{*}: H^{1}(X, N) \rightarrow H^{1}(X, W)$ induced by $p$. Fix $[b] \in H^{1}(X, N)$; our goal is to show that $[b]$ lifts to $H^{1}(X, S)$.

3.2. Lemma. Let $[a]=p_{*}([b]) \in H^{1}(X, W)$. Then

$$
[a] \in \operatorname{Im}\left(H^{1}(X, S) \stackrel{q_{*}}{\rightarrow} H^{1}(X, W)\right) .
$$

Proof of Lemma 3.2. The obstruction to lifting $[a]$ to $H^{1}(X, S)$ is the class

$$
\Delta([a]) \in H^{2}\left(X,{ }_{a} \mu\right),
$$

where ${ }_{a} \mu$ denotes the group $\mu$ twisted by the torsor $a$ [Gir, IV.4.2.8]. We now use the commutative diagram

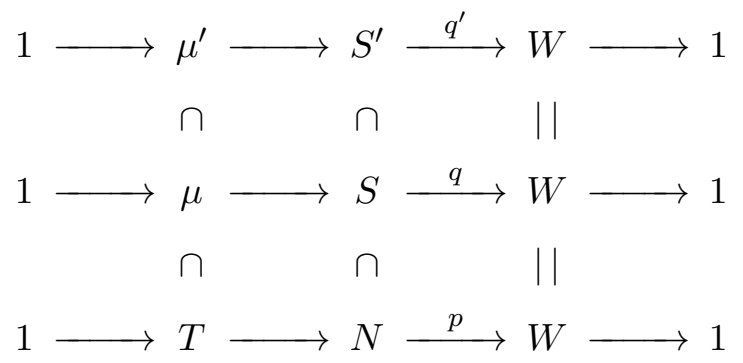

with exact rows and the functoriality of the obstruction $\Delta([a])$. If $\Delta^{\prime}([a]) \in$ $H^{2}\left(X,{ }_{a} \mu^{\prime}\right)$ is the obstruction to lifting $[a]$ to $H^{1}\left(X, S^{\prime}\right)$, via $q_{*}^{\prime}: H^{1}\left(X, S^{\prime}\right) \rightarrow$ $H^{1}(X, W)$, then $\Delta([a])$ is the image of $\Delta^{\prime}([a])$ under the natural map $H^{2}\left(X,{ }_{a} \mu^{\prime}\right) \rightarrow$ $H^{2}\left(X,{ }_{a} \mu\right)$.

The commutative diagram

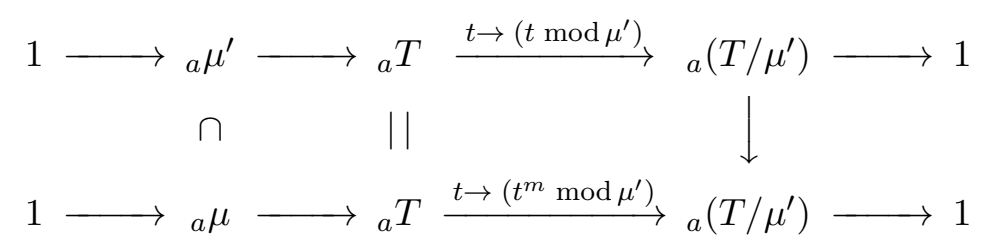

with exact rows gives rise to the commutative exact diagram

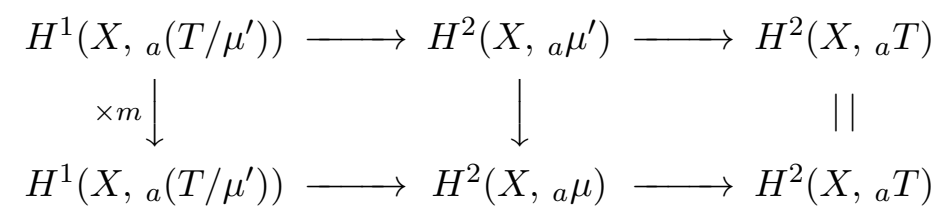


which we will now analyse. Recall that the middle vertical map sends $\Delta^{\prime}([a]) \in H^{2}\left(X,{ }_{a} \mu^{\prime}\right)$ to $\Delta([a]) \in H^{2}\left(X,{ }_{a} \mu\right)$. Since we are given that $[a]$ lifts to $[b] \in H^{1}(X, N)$, we have

$$
\Delta^{\prime}([a]) \in \operatorname{ker}\left(H^{2}\left(X,{ }_{a} \mu^{\prime}\right) \rightarrow H^{2}\left(X,{ }_{a} T\right)\right)
$$

and thus

$$
\Delta^{\prime}([a]) \in \operatorname{Im}\left(H^{1}\left(X,{ }_{a}\left(T / \mu^{\prime}\right)\right) \rightarrow H^{2}\left(X,{ }_{a} \mu^{\prime}\right)\right) .
$$

In order to prove the lemma (i.e., to prove that $\Delta([a])=0$ ), it now suffices to show that the vertical map

$$
\begin{aligned}
& H^{1}\left(X,{ }_{a}\left(T / \mu^{\prime}\right)\right) \\
& \times m \downarrow \\
& H^{1}\left(X,{ }_{a}\left(T / \mu^{\prime}\right)\right)
\end{aligned}
$$

in the above diagram is trivial.

If $p: X^{\prime} \rightarrow X$ is a cover (i.e., a finite étale map) of degree $m$ and $H$ is a commutative affine $X$-group scheme, we will denote the trace morphism by $N_{X^{\prime} / X}: \mathrm{R}_{X^{\prime} / X}(H) \rightarrow H$; cf. [CTS, 0.4]. If $p$ has degree $m$, the composition

$$
H \longrightarrow \mathrm{R}_{X^{\prime} / X}(H) \stackrel{N_{X^{\prime} / X}}{\longrightarrow} H
$$

of $N_{X^{\prime} / X}$ with the natural map $H \rightarrow \mathrm{R}_{X^{\prime} / X}(H)$ is multiplication by $m$.

Now let $Y \rightarrow X$ be the $W$-torsor associated to $a$ and apply the above facts to the generalized Galois covering $X^{\prime}=Y \times_{k} k^{\prime} \rightarrow X$ of degree $m=n d$, with $H={ }_{a}\left(T / \mu^{\prime}\right)$. Note that this covering trivializes $a$ and splits $T$. The map (3.5) can be decomposed as

$$
H^{1}\left(X,{ }_{a} T\right) \longrightarrow H^{1}\left(X, \mathrm{R}_{X^{\prime} / X}\left({ }_{a}\left(T / \mu^{\prime}\right)\right)\right) \longrightarrow H^{1}\left(X,{ }_{a}\left(T / \mu^{\prime}\right)\right) .
$$

Shapiro's lemma and condition (1.1) imply that

$$
H^{1}\left(X, \mathrm{R}_{X^{\prime} / X}\left({ }_{a}\left(T / \mu^{\prime}\right)\right)\right)=H^{1}\left(X^{\prime},{ }_{a}\left(T / \mu^{\prime}\right)\right)=\operatorname{Pic}\left(X^{\prime}\right)^{\operatorname{rank}(T)}=0 .
$$

Hence the map (3.5) is trivial, as claimed. The proof of Lemma 3.2 is now complete.

We are now ready to finish the proof of Proposition 3.1. Let $[c] \in$ $H^{1}(X, S)$ be such that $q_{*}([c])=[a]$. The bottom two rows of (3.3) give rise to the diagram

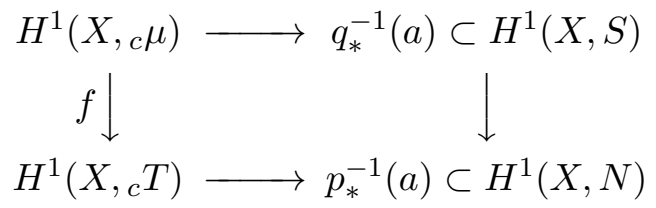

where the horizontal arrows are the "torsion" maps (see $\S 2$ ). Recall that our goal is to show that $[b] \in p_{*}^{-1}([a]) \subset H^{1}(X, N)$ lies in the image of $H^{1}(X, S)$. 
If $X=\operatorname{Spec}(K)$ for some field $K / k$ then a twisting argument [Se, I.5.5] shows that the map

$$
H^{1}\left(K,{ }_{c} T\right) \rightarrow p_{*}^{-1}([a])
$$

is surjective. The same twisting argument goes through for any $k$-scheme $X$ Gir, III.3.2.4]; in this case we can also conclude that the map

$$
H^{1}\left(X,{ }_{c} T\right) \rightarrow p_{*}^{-1}([a])
$$

is surjective. Thus it suffices to prove that the vertical map $f$ in the above diagram is surjective as well. The exact sequence

$$
1 \longrightarrow{ }_{c} \mu \longrightarrow{ }_{c} T \stackrel{t \rightarrow\left(t^{m} \bmod \mu^{\prime}\right)}{\longrightarrow}{ }_{c} T / \mu^{\prime} \longrightarrow 1
$$

gives rise to the exact sequence

$$
H^{1}\left(X,{ }_{c} \mu\right) \stackrel{f}{\longrightarrow} H^{1}\left(X,{ }_{c} T\right) \longrightarrow H^{1}\left(X,{ }_{c}(T / \mu)\right) .
$$

It thus remains to show that the map

$$
H^{1}\left(X,{ }_{c} T\right) \rightarrow H^{1}\left(X,{ }_{c}(T / \mu)\right)
$$

in this sequence is trivial. Indeed, since the group homomorphism

$$
{ }_{c} T \rightarrow{ }_{c}\left(T / \mu^{\prime}\right)
$$

in (3.6) factors through

$$
\times m:{ }_{c}\left(T / \mu^{\prime}\right) \stackrel{\times m}{\longrightarrow}{ }_{c}\left(T / \mu^{\prime}\right),
$$

the map (3.7) factors through

$$
\times m: H^{1}\left(X,{ }_{c}\left(T / \mu^{\prime}\right)\right) \longrightarrow H^{1}\left(X,{ }_{c}\left(T / \mu^{\prime}\right)\right)
$$

which we showed to be trivial at the end of the proof of Lemma 3.2. We conclude that the map (3.7) is trivial, as claimed. This completes the proof of Proposition 3.1 .

3.8. Remark. Let $k$ be a ring, $T$ is a maximal $k$-torus in an affine algebraic $k$-group $G$ and $N=N_{G}(T)$. This is a natural setting, where Proposition 3.1 can be applied. However, it is not a priori clear for which $G$ one can construct a finite group $S^{\prime}$ as in Proposition 3.1. In fact, it is not even clear in general which affine $k$-groups $G$ contain a maximal $k$-torus $T$. If we can find a maximal $k$-torus $T \subset G$ and a finite $k$-subgroup $S^{\prime} \subset N=N_{G}(T)$ with desired properties, we would also like to know under what circumstances one can conclude that the map $H^{1}(X, S) \rightarrow H^{1}(X, G)$ is surjective. In the sequel we will give partial answers to these questions, under additional assumptions on $k$. 


\section{Proof of Theorem 1.2(A) And (B)}

(a) Let $T$ be a maximal $k$-torus of $G$ and $N=N_{G}(T)$. Since

$$
N^{0} \subset\left(N_{G^{0}}(T)\right)^{0}=T \subset N,
$$

we have $N^{0}=T$. Hence, $N$ is smooth and $W$ is a finite constant group. Let $p: N \rightarrow W=N / T$ be the natural projection. By Proposition 3.1 it suffices to construct a finite $k$-subgroup $S^{\prime} \subset N$ such that $p\left(S^{\prime}\right)=W$. In fact, we will construct $S^{\prime}$ so that $\mu^{\prime}$ be the $n$-torsion subgroup of $T$, where $n=|W|$.

Consider the exact sequences

$$
1 \rightarrow T \rightarrow N \stackrel{p}{\rightarrow} W \rightarrow 1 \quad \text { and } \quad 1 \rightarrow \mu^{\prime} \rightarrow T \stackrel{\times n}{\rightarrow} T \rightarrow 1 .
$$

According to [DG, II.2, Proposition 2.3] (cf. also [SGA3, XVII, App. I.3.1, page 622]), extensions of $W$ by $T$ are classified by the Hochschild cohomology group $H_{0}^{2}(W, T)$. Since $W$ is a constant group scheme, $H_{0}^{2}(W, T)$ is isomorphic to the usual cohomology group $H^{2}(W, T(k))$; see DG, III.6.4, Proposition 4.2]. Thus the first sequence yields a class in $H^{2}(W, T(k))$. Since $n \cdot H^{2}(W, T(k))=0$, the second sequence tells us that this class comes from $H^{2}\left(W, \mu^{\prime}(k)\right)$. In other words, there is an extension $S^{\prime} \subset N$ of $W$ by $\mu^{\prime}$ such that $N$ is the push-out of $S^{\prime}$ by the morphism $\mu^{\prime} \hookrightarrow T$. This completes the construction of $S^{\prime}$.

(b) Let $T$ be a maximal split torus of $G$ defined over $\mathbb{Z}$. Note that $W=$ $N(T) / T$ is a constant finite group scheme; this follows from the fact that $W$ is representable by a $\mathbb{Z}$-group scheme which is finite étale [SGA3, XII.2.1.b].

It remains to construct a finite subgroup $S^{\prime} \subset N$ which surjects onto $W$; the desired conclusion will then follow from Proposition 3.1 .

Our construction of $S^{\prime}$ will be based on schematic adherence, which associates to a closed $\mathbb{Q}$-subscheme $V \subset G_{\mathbb{Q}}$ its Zariski closure $\widetilde{V}$ in $G_{\mathbb{Z}}$. Schematic adherence induces a one-to-one correspondence between $\mathbb{Q}$-subchemes of $G_{\mathbb{Q}}$ and flat closed $\mathbb{Z}$-subchemes of $G_{\mathbb{Z}}[\mathbb{B T}$, I.2.6]. In particular, it maps $\mathbb{Q}$-subgroups of $G_{\mathbb{Q}}$ into flat $\mathbb{Z}$-group subschemes of $G$ [BT, I.2.7] (see also [GM, §3]).

Let $n=|W|$ and $T=\mathbb{G}_{m}^{r}$, where $r$ is the rank of $G$. As pointed out by Tits [T], the fact that $H^{1}(\mathbb{Z}, T)=\operatorname{Pic}(\mathbb{Z})^{r}=0$ implies that the sequence

$$
0 \rightarrow T(\mathbb{Z}) \rightarrow N(\mathbb{Z}) \rightarrow W \rightarrow 1 .
$$

is exact. Since $T(\mathbb{Z})=\{ \pm 1\}^{r}, N(\mathbb{Z})$ is a finite group. View $N(\mathbb{Z})$ as a finite constant $\mathbb{Q}$-subgroup of $G_{\mathbb{Q}}$ and let $S^{\prime}$ be its schematic adherence in $N / \mathbb{Z}$. Then $S^{\prime}$ is a finite flat $\mathbb{Z}$-subgroup scheme of $N$. Since $N(\mathbb{Z})$ surjects onto $W$, so does $S^{\prime}$.

4.1. Remark. In the case where $X=\operatorname{Spec}(K)$ for some field $K / k$, Theorem [1.2(a) reduces to CGR, Theorem 1.1(a)], and our proof proceeds along similar lines. Note however, that there is a small mistake in the proof of CGR, Theorem 1.1(a)]. On page 565 in CGR, in the setting of Lemma 3.2 above, we said that the obstruction $\Delta(a)$ (denoted by $\delta([a]$ ) 
there) to lifting $a$ to $H^{1}(X, S)$ lies in $H^{2}(X, \mu)$, instead of $H^{2}\left(X,{ }_{a} \mu\right)$. This mistake is corrected in the proof of Lemma 3.2 in the present paper. As a consequence, the (corrected) argument in this paper is a bit longer than in [CGR], and the group $S$ is a bit larger; here $S \cap T={ }_{n^{2}} T$, where as in CGR] $S \cap T={ }_{n} T$.

\section{TORAL TORSORS AND A THEOREM OF GRothendiECK}

Let $X$ be a scheme and $G$ be a smooth affine group scheme over $X$. Assume that the connected component $G^{0}$ is reductive. We say that a $G$ torsor $E$ over $X$ is toral if the twisted $X$-group scheme ${ }^{E} G$ admits a maximal torus defined over $X$. We denote by $H_{\text {toral }}^{1}(X, G) \subset H^{1}(X, G)$ the set of toral classes. The following lemma is well known.

5.1. Lemma. Assume that $G^{0} / X$ admits a maximal $X$-torus $T$. Then

$$
H_{\text {toral }}^{1}(X, G)=\operatorname{Im}\left(H^{1}\left(X, N_{G}(T)\right) \rightarrow H^{1}(X, G)\right) .
$$

Proof. Let $E / X$ be a $G$-torsor. The functor $\mathcal{T} / X$ of maximal tori of ${ }^{E} G$ is representable by a separated smooth scheme $\Sigma$ of finite type over $X$ [SGA3, XII.1.10]. In fact, $\Sigma$ is the $E$-twist of homogeneous space $G / N_{G}(T)$ (whose points represent maximal tori in $G$ ); equivalently, $\Sigma$ can be thought of as the quotient $E / N_{G}(T)$ (see [SGA3, XXIV.4.2.1]). So the following are equivalent:

(1) ${ }^{E} G$ has a maximal $X$-torus,

(2) $\mathcal{T}(X) \neq \emptyset$,

(3) $\left(E / N_{G}(T)\right)(X) \neq \emptyset$.

By [DG, III, $\S 4$, Prop. 4.6], condition (3) is equivalent to

$$
[E] \in \operatorname{Im}\left(H^{1}\left(X, N_{G}(T)\right) \rightarrow H^{1}(X, G)\right),
$$

and the lemma follows.

The following theorem of Grothendieck tells us that if $G$ is a reductive group scheme over a semilocal ring $k$ then every $G$-torsor over $k$ is toral.

5.2. Theorem. ([SGA3, XIV.3.20]). Let $G$ be a reductive group scheme defined over a semilocal ring $k$. Then $G$ admits a maximal $k$-torus $T$.

The corollary below will be of particular interest to us in the sequel.

5.3. Corollary. Let $G$ be a smooth affine reductive groups scheme defined over a semilocal ring $k$. Suppose $T$ is a maximal $k$-torus of $G$. Then the natural map $H^{1}\left(R, N_{G}(T)\right) \rightarrow H^{1}(R, G)$ is surjective for any semilocal ring $R / k$.

Proof. By Theorem 5.2 every $G$-torsor over $\operatorname{Spec}(R)$ is toral. That is,

$$
H^{1}(R, G)_{\text {toral }}=H^{1}(R, G) .
$$

The corollary now follows from Lemma 5.1. 


\section{Proof of Theorem 1.2(c)}

Throughout this section $k$ will denote a semilocal ring and $G$ an affine connected reductive group scheme defined over $k$. Suppose that the radical torus of $G$ is isotrivial. We will now proceed to prove Theorem 1.2(c) in four steps.

Case 1. $G$ split, semisimple and adjoint. That is, $G=G_{0} \times_{\mathbb{Z}} k$, where $G_{0}$ is an adjoint split group defined over $\mathbb{Z}$. Let $T_{0}$ be a maximal split torus in $G_{0}$ defined over $\mathbb{Z}$ and let $S_{0}^{\prime} \subset N_{G_{0}}\left(T_{0}\right)$ be the finite subgroup satisfying the conditions of Proposition 3.1 constructed in the previous section. Then $S^{\prime}=S_{0}^{\prime} \otimes_{\mathbb{Z}} k$ satisfies the same conditions in $G$, relative to the maximal torus $T=T_{0} \otimes_{\mathbb{Z}} k$ of $G$. The desired conclusion now follows from Proposition 3.1,

Case 2. $G$ is a quasi-split semisimple and adjoint. In this case $G$ is $k$-isomorphic to the twist ${ }_{h_{*}(a)}\left(G_{1}\right)$, where $G_{1}$ is a split adjoint group scheme over $k$ of the same type as $G$,

$$
a \in Z_{e ́ t}^{1}\left(k, \operatorname{Aut}_{\text {Dyn }}(D)\right),
$$

and $D$ is the Dynkin scheme of $G_{1}$, relative to a maximal split $k$-torus $T_{1} \subset G_{1}$; see 92 . The cocycle $h_{*}(a)$ preserves the maximal torus $T_{1}$ and the finite subgroup of $N_{G_{1}}\left(T_{1}\right)$ constructed in Case 1 . In Case 1 we called this finite subgroup $S^{\prime}$; now we will denote it by $S_{1}^{\prime}$. Recall that $S_{1}^{\prime}$ satisfies the conditions of Proposition 3.1 relative to $T_{1}$; that is, $S_{1}^{\prime}$ normalizes $T_{1}$ and projects surjectively onto $W_{1}=N_{G_{1}}\left(T_{1}\right) / T_{1}$. Now observe that the $\operatorname{group}_{h_{*}(a)}\left(S_{1}^{\prime}\right)$ satisfies the same conditions in $G={ }_{h_{*}(a)}\left(G_{1}\right)$, relative to the maximal $k$-torus $h_{*}(a)\left(T_{1}\right)$. The desired conclusion now follows from Proposition 3.1 .

Case 3: $G$ is semisimple and adjoint. In this case $G$ is $k$-twisted form of a Chevalley group $G_{0}$ [SGA3, XXIII.5.7]. In other words, there exists a cocycle $b \in Z^{1}\left(k, \operatorname{Aut}\left(G_{0}\right)\right)$ such that $G \cong{ }_{b} G_{0}$. Let $a$ be the image of $b$ under the projection

$$
Z_{f p p f}^{1}\left(k, \operatorname{Aut}\left(G_{0}\right)\right) \rightarrow Z_{f p p f}^{1}(k, \operatorname{Aut}(D)),
$$

where $D$ is the Dynkin scheme of $G_{0}$. Consider the following commutative exact diagram of pointed sets

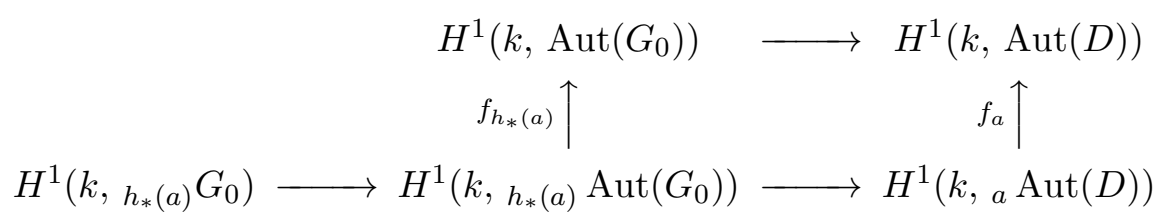

where $f_{h_{*}(a)}$ and $f_{a}$ stand for the "torsion" bijections; see $\oint 2$, By a diagram chase, there exists $[c] \in H^{1}\left(k, h_{*}(a) G_{0}\right)$ such that $G$ is isomorphic to the twisted group ${ }_{c}\left(h_{*}(a) G_{0}\right)$, i.e., $G$ is a $k$-inner form of the quasi-split group $h_{*}(a) G_{0}$. 
By Case 2 we know that Theorem 1.2(c) holds for $G_{1}={ }_{h_{*}(a)} G_{0}$. That is, there exists a maximal torus $T_{1} \subset G_{1}$ defined over $k$ and a finite $k$ subgroup $S_{1} \subset N_{G}\left(T_{1}\right)$, such that $S_{1}$ is an extension of a twisted constant group scheme by a finite $k$-group of multiplicative type and the natural map $H^{1}\left(k, S_{1}\right) \longrightarrow H^{1}\left(k, N_{G_{1}}\left(T_{1}\right)\right)$ is surjective. Moreover, by Corollary 5.3 the map $H^{1}\left(k, N_{G_{1}}\left(T_{1}\right)\right) \rightarrow H^{1}\left(k, G_{1}\right)$ is also surjective. We conclude that the map $H^{1}\left(k, S_{1}\right) \rightarrow H^{1}\left(k, G_{1}\right)$ is surjective. We may thus assume that $c$ takes values in $S_{1}$.

Now set $S:={ }_{c} S_{1}$. Then $S$ embeds in ${ }_{c} T_{1}$. Consider the diagram

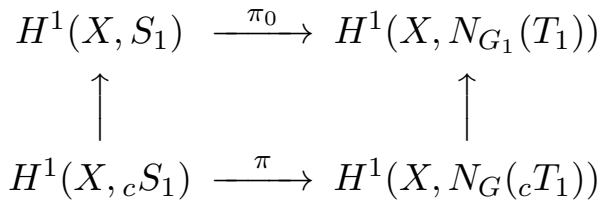

where the vertical arrows are the "torsion" bijections; see $\sqrt{2}$. Since $\pi_{0}$ is surjective, so is $\pi$.

Case 4. $G$ is reductive and the radical torus $C=\operatorname{rad}(G)$ is isotrivial. Consider the semisimple $k$-group $H=[G, G]$. Let $Z$ be the center of $H$, $G^{\prime}=G / Z$ and $f: G \rightarrow G^{\prime}$ be the natural projection. Then $G^{\prime} \simeq C^{\prime} \times H^{\prime}$, where $C^{\prime}=C / C \cap H$ and $H^{\prime}=H / Z$ is an adjoint semisimple group. Since we are assuming that $C$ is an isotrivial $k$-torus, there exists a finite étale surjective covering $\widetilde{k} / k$ which splits $C$. Note that $\widetilde{k}$ is a semilocal ring and $C^{\prime} \times{ }_{k} \widetilde{k}$ is also a split torus.

Let $m$ be the degree of the covering $\widetilde{k} / k$ and let $\mu$ be the $m$-torsion subgroup of $C^{\prime}$. Note that the canonical mapping $H^{1}(k, \mu) \rightarrow H^{1}\left(k, C^{\prime}\right)$ is surjective. Indeed, the restriction-corestriction formula [CTS, 0.4]

$$
\times m=\operatorname{Cor}_{k}^{\widetilde{k}} \circ \operatorname{Res}_{k}^{\widetilde{k}}: H^{1}\left(k, C^{\prime}\right) \rightarrow H^{1}\left(k, C^{\prime}\right)
$$

together with the fact that $H^{1}\left(\widetilde{k}, C^{\prime}\right)=0$ (Hilbert's Theorem 90) imply that the map

$$
\times m: H^{1}\left(k, C^{\prime}\right) \rightarrow H^{1}\left(k, C^{\prime}\right)
$$

is trivial.

Let $T^{\prime}$ and $S^{\prime} \subset N_{H^{\prime}}\left(T^{\prime}\right)$ be the subgroups constructed in Case 3 for $H^{\prime}$ and let $X / k$ be a scheme satisfying condition (1.1). Then the canonical morphism $\pi^{\prime}: H^{1}\left(X, \mu \times S^{\prime}\right) \rightarrow H^{1}\left(X, N_{G^{\prime}}\left(T^{\prime}\right)\right)$ is surjective. We claim that $S=f^{-1}\left(\mu \times S^{\prime}\right)$ is as required, i.e. $H^{1}(X, S) \rightarrow H^{1}\left(X, N_{G}(T)\right)$ is surjective where $T=f^{-1}\left(C^{\prime} \times T^{\prime}\right)$.

Indeed, the exact sequences $1 \rightarrow Z \rightarrow N_{G}(T) \rightarrow N_{G^{\prime}}\left(T^{\prime}\right) \rightarrow 1$ and $1 \rightarrow Z \rightarrow S \rightarrow S^{\prime} \rightarrow 1$ give rise to a commutative diagram

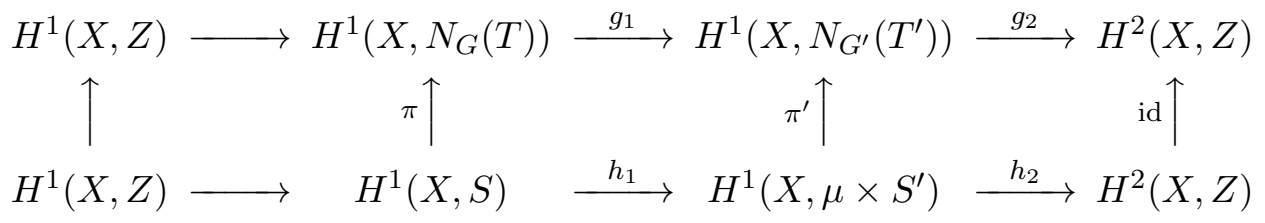


Here $g_{2}, h_{2}$ are connecting homomorphisms [Gir, IV.4.3.4]. Fix an element $[a] \in H^{1}\left(X, N_{G}(T)\right)$ and let $[b]=g_{1}([a])$. Since $\pi^{\prime}$ is surjective, there is a class $[c] \in H^{1}\left(X, \mu \times S^{\prime}\right)$ such that $\pi^{\prime}([c])=[b]$. Since $h_{2}([c])=g_{2} \pi^{\prime}([c])=$ 0 , there is $[d] \in H^{1}(X, S)$ such that $h_{1}([d])=[c]$. Thus the classes $[a]$ and $\pi([d])$ have the same image in $H^{1}\left(X, N_{G^{\prime}}\left(T^{\prime}\right)\right)$. A twisting argument shows that the map $H^{1}\left(X,{ }_{d} Z\right) \rightarrow g_{1}^{-1}\left(g_{1}([a])\right)$ is surjective; see [Gir, III.3.2.4]. Since $Z \subset S$, we have ${ }_{d} Z \subset{ }_{d} S$ implying $[a] \in \operatorname{Im} \pi$. This completes the proof of Theorem 1.2 (c).

\section{Proof of Theorem 1.3 and an Application}

Theorem 1.3 stated in the introduction is an easy consequence of Theorem 1.2(c) and Corollary 5.3. Indeed, choose $T$ and $S$ as in Theorem 1.2(c) and let $R$ be a semilocal ring containing $k$. Since $X=\operatorname{Spec}(R)$ satisfies condition (1.1), Theorem 1.2(c) tells us that the natural map $H^{1}(R, S) \rightarrow$ $H^{1}(R, N(T))$ is surjective. By Corollary 5.3 the map $H^{1}\left(R, N_{G}(T)\right) \rightarrow$ $H^{1}(R, G)$ is also surjective, and Theorem 1.3 follows.

We will now discuss an application of Theorem 1.3 . Let $G$ be a linear algebraic group defined over a field $k$ and $\pi: Y \rightarrow X$ be a $G$-torsor over a $k$-scheme $X$. As usual, we will say that $\pi$ admits reduction of structure to a $k$-subgroup $S \subset G$ if the class in $H^{1}(X, G)$ represented by $\pi$ lies in the image of the natural map $H^{1}(X, S) \rightarrow H^{1}(X, G)$. Equivalently, $\pi$ admits reduction of structure to $S$ if there exists a $G$-equivariant morphism $Y \rightarrow G_{X} / S_{X}$.

Suppose $U \rightarrow X$ is a morphism and $Y_{U}$ is the pull-back of $Y$ to $U$ :

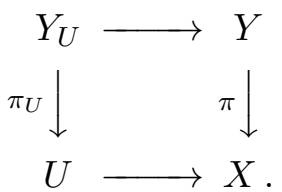

We say that $Y$ admits reduction of structure to $S$ over $U$ if the $G$-torsor $\pi_{U}: Y_{U} \rightarrow U$ admits reduction of structure to $S$.

7.1. Proposition. Let $G$ be a linear algebraic group defined over a field $k$. Assume that the connected component $G^{0}$ is reductive and either $G$ is connected or $k$ is algebraically closed. Then there exists a $k$-subgroup $S \subset G$ with the following property. For any $G$-torsor $\pi: Y \rightarrow X$ over an affine $k$-scheme $X$ and any finite collection of (not necessarily closed) points $x_{1}, \ldots, x_{n} \in X$, there exists an open subscheme $U \subset X$ containing $x_{1}, \ldots, x_{n}$ such that $\pi$ admits reduction of structure to $S$ over $U$.

Proof. Let $R=\mathcal{O}_{x_{1}, \ldots, x_{n}}$ be the semilocal ring of $X$ at $x_{1}, \ldots, x_{n}$. By Theorem $1.3 \pi$ admits reduction of structure to $S$ over $\operatorname{Spec}(R) \subset X$. That is, there exists a $G$-equivariant morphism $\phi: Y_{R} \rightarrow G_{R} / S_{R}$.

Since $R$ is, by definition, the direct limit of $\mathcal{O}_{X}(U)$, as $U$ ranges over the open subsets of $X$ containing $x_{1}, \ldots, x_{n}, \phi$ extends over some open subscheme $X_{0}$ of $X$ containing $x_{1}, \ldots, x_{n}$. In other words, $\pi$ admits reduction of structure to $S$ over $X_{0}$. 


\section{TORSORS ON AFFINE SPACES}

Let $k$ be a field of characteristic 0 , and $\bar{k}$ be an algebraic closure of $k$. In this section we will apply Theorem 1.2(c) in the case where $X$ is the affine space $\mathbb{A}_{k}^{n}$. The key observation here is that $\mathbb{A}_{k}^{n}$ is simply connected. By the fundamental exact sequence for $\pi_{1}$ [SGA1, IX.6.1], we have an isomorphism

$$
\pi_{1}\left(\mathbb{A}_{k}^{n}, \overline{0}\right) \stackrel{\sim}{\longrightarrow} \operatorname{Gal}(\bar{k} / k),
$$

where $\pi_{1}\left(\mathbb{A}_{k}^{n}, \overline{0}\right)$ stands for the algebraic fundamental group of $\mathbb{A}_{k}^{n}$ relative to the base point $\overline{0}: \operatorname{Spec}(\bar{k}) \rightarrow \mathbb{A}_{k}^{n}$. In other words, every finite étale cover of $\mathbb{A}_{k}^{n}$ is of the form $\mathbb{A}_{K}^{n}$, where $K / k$ is an étale $k$-algebra. Since $\operatorname{Pic}\left(\mathbb{A}_{K}^{n}\right)=0$, this implies that $X=\mathbb{A}_{k}^{n}$ satisfies condition (1.1).

8.1. Proposition. Let $k$ be a field of characteristic zero, $n \geq 0$ be an integer, and $G$ be a (connected) reductive group over $k$. Then

$$
H^{1}(k, G) \stackrel{\sim}{\longrightarrow} H^{1}\left(\mathbb{A}_{k}^{n}, G\right)_{\text {toral }} .
$$

In other words, every toral torsor on $\mathbb{A}_{k}^{n}$ is constant.

Proof. Since $k$ is a field, Theorem 1.2(c) applies to $G$. Let $S \subset G$ be the finite $k$-subgroup as in Theorem 1.2. As we noted before the statement of the proposition, $X=\mathbb{A}_{k}^{n}$ satisfies condition (1.1). Thus the natural map $H^{1}\left(\mathbb{A}_{k}^{n}, S\right) \rightarrow H^{1}\left(\mathbb{A}_{k}^{n}, N(T)\right)$ is surjective. By Lemma 5.1, the map

$$
H^{1}\left(\mathbb{A}_{k}^{n}, S\right) \rightarrow H^{1}\left(\mathbb{A}_{k}^{n}, G\right)_{\text {toral }}
$$

is also surjective. The kernel of the natural map $H^{1}\left(\mathbb{A}_{k}^{n}, S\right) \rightarrow H^{1}\left(\mathbb{A}_{\frac{n}{k}}, S\right)$ consists of those $S$-torsors on $\mathbb{A}_{k}^{n}$ which become trivial on $\mathbb{A}_{\frac{k}{n}}^{n}$. Since $\mathbb{A}_{\bar{k}} \rightarrow \mathbb{A}_{k}$ is a Galois cover, with Galois group $\operatorname{Gal}(\bar{k} / k)$, this kernel is $H^{1}\left(k, S\left(\mathbb{A}_{\bar{k}} \frac{n}{}\right)\right)$, where $H^{1}$ stands for Galois cohomology. Since $S\left(\mathbb{A}_{\bar{k}} \frac{n}{}\right)=$ $S(\bar{k})$, this yields an exact sequence

$$
1 \rightarrow H^{1}(k, S(\bar{k})) \rightarrow H^{1}\left(\mathbb{A}_{k}^{n}, S\right) \rightarrow H^{1}\left(\mathbb{A}_{k}^{n}, S\right) .
$$

Since $\mathbb{A}_{\frac{k}{k}}$ is simply connected, $H^{1}\left(\mathbb{A}_{\frac{k}{k}}, S\right)=1$, and hence the map

$$
H^{1}(k, S(\bar{k})) \rightarrow H^{1}\left(\mathbb{A}_{k}^{n}, S\right)
$$

is surjective. The commutative exact diagram of pointed sets

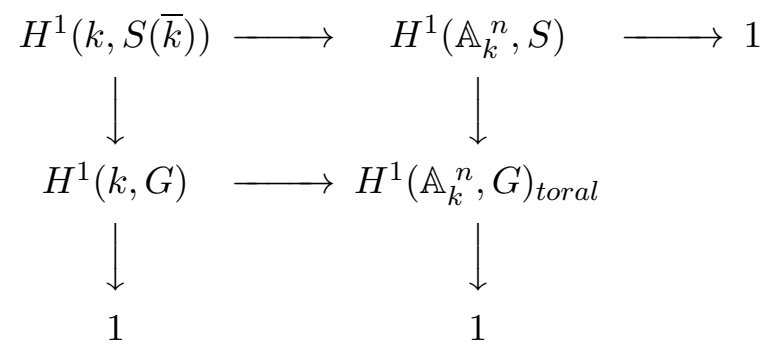

shows that the natural map $H^{1}(k, G) \rightarrow H^{1}\left(\mathbb{A}_{k}^{n}, G\right)_{\text {toral }}$ is surjective. This map is also injective. Indeed, suppose $G$-torsors $T_{1} \rightarrow \operatorname{Spec}(k)$ and $T_{2} \rightarrow$ 
$\operatorname{Spec}(k)$ map to the same $G$-torsor $Y \rightarrow \mathbb{A}_{k}^{n}$, i.e., $Y \simeq T_{i} \times_{\operatorname{Spec}(k)} \mathbb{A}_{k}^{n}$ for $i=1,2$. Then both $T_{1}$ and $T_{2}$ are isomorphic to the fiber of $Y$ over $0 \in \mathbb{A}_{k}^{n}$. Hence, $T_{1}$ and $T_{2}$ represent the same class in $H^{1}(k, G)$. We conclude that the map $H^{1}(k, G) \rightarrow H^{1}\left(\mathbb{A}_{k}^{n}, G\right)_{\text {toral }}$ is an isomorphism.

8.2. Remark. There are examples of non constant $G$-torsors $P$ over affine spaces; see Ojanguren-Sridharan [OS] (cf. also [K, VII.10]). Proposition 8.1 tells us that in these examples the twisted groups ${ }^{P} G$ do not carry maximal tori.

8.3. Remark. As we pointed out in the introduction, the scheme

$$
X=\operatorname{Spec}\left(k\left[x_{1}^{ \pm 1}, \ldots, x_{n}^{ \pm 1}\right]\right)
$$

also satisfies condition (1.1) (in characteristic zero), so in this case the map $H^{1}(X, S) \rightarrow H^{1}(X, G)_{t o r a l}$ is also surjective. This fact is used in [GP].

\section{REFERENCES}

[BT] F. Bruhat, J. Tits, Groupes réductifs sur un corps local. II. Schémas en groupes. Existence d'une donnée radicielle valuée, Inst. Hautes Etudes Sci. Publ. Math. 60 (1984), 197-376.

[CGP] V. Chernousov, P. Gille, A. Pianzola, Torsors on the affine line and on the punctured line, in preparation.

[CGR] V. Chernousov, P. Gille, Z. Reichstein, Resolving G-torsors by abelian base extensions, J. Algebra 296 (2006), 561-581.

[CTS] J.-L. Colliot-Thélène and J.-J. Sansuc, Principal homogeneous spaces under flasque tori: applications, J. Algebra 106 (1987), 148-205.

[DG] M. Demazure, P. Gabriel, Groupes algébriques, I, North-Holland, 1970.

[GM] P. Gille, L. Moret-Bailly, Actions algébriques de groupes arithmétiques, preprint (2006), available on the authors' home pages.

[GP] P. Gille and A. Pianzola, Galois cohomology and forms of algebras over Laurent polynomial rings $I I$, in preparation.

[GR] P. Gille and Z. Reichstein, Lower bounds for the essential dimension of linear algebraic groups, to appear in Commentarii Math. Helv.

[Gir] J. Giraud, Cohomologie non-abélienne, Springer, 1970.

[K] M. A. Knus, Quadratic and hermitian forms over rings, Grundlehren der mat. Wissenschaften 294, Springer, 1991.

[M] J. S. Milne, Étale Cohomology, Princeton Mathematical Series, 33, Princeton University Press, 1980.

[OS] M. Ojanguren, R. Sridharan, Cancellation of Azumaya algebras, J. Algebra 18 (1971), 501-505.

[SGA1] Séminaire de Géométrie algébrique de l'I.H.E.S., Revêtements étales et groupe fondamental, dirigé par A. Grothendieck, Lecture Notes in Math. 224. Springer, 1971.

[SGA3] Séminaire de Géométrie algébrique de l'I.H.E.S., 1963-1964, schémas en groupes, dirigé par M. Demazure et A. Grothendieck, Lecture Notes in Math. 151-153. Springer, 1970.

[Se] J.-P. Serre, Galois Cohomology, Springer, 1997.

[T] J. Tits, Normalisateurs de tores. I. Groupes de Coxeter étendus, J. Algebra 4 (1966), 96-116. 
Department of Mathematics, University of Alberta, Edmonton, Alberta T6G 2G1, CANADA

E-mail address: chernous@math.ualberta.ca

UMR 8552 Du CNRS, DMA, Ecole nORMAle SupÉrieure, F-75005 PARIS, France E-mail address: Philippe.Gille@ens.fr

Department of Mathematics, University of British Columbia, Vancouver, BC V6T 1Z2, CANADA

E-mail address: reichst@math.ubc.ca

$U R L$ : www.math.ubc.ca/ $\sim$ reichst 